\title{
Correlation of gammadelta-T-cells, Th17 cells and IL- 17 in peripheral blood of patients with allergic rhinitis
}

\author{
Huang Xuekun, Yang Qintai, Chen Yulian and Zhang Gehua
}

\section{Summary}

Background: Gammadelta-T-cells play an important role in the mucosal immune system of the respiratory tract. Th17 cells plays an important role in promoting inflammation and autoimmune diseases. Th17 cells mainly secrete IL-17. However, little information is available on the relation between gammadelta-T-cells and Th17 cells in allergic rhinitis (AR).

Objective: To explore the correlation between the prevalence of gammadelta-T-cells, the prevalence of Th17 cells and the expression of IL-17 in the peripheral blood of patients with AR.

Methods: Peripheral blood from the AR group (32 cases) and the control group ( 20 cases) was collected from March 2012 to July 2012. The percentages of gammadelta-T-cells and Th17 cells were measured by flow cytometry, and the levels of IL-17 were measured by ELISA.

Results: The percentage of gammadeta-T-cells and Th17 cells in the AR group were significantly higher than those in the control group $(P<0.01)$. The levels of IL-17 in the AR group were also significantly higher than those in the control group $(P<0.01)$. There were positive correlations between the gammadelta-T-cell percentage and the Th17 cell percentage in the peripheral blood of AR patients $(r=0.597, P<0.01)$; and between the gammadelta-T-cells percentage and the levels of IL-17 $(r=0.469, P<0.01)$. However, no correlation was found between the Th17 cell percentage and the levels of IL-17 $(r=0.100, P>0.05)$.

From Department of Otolaryngology, The Third Affiliated Hospital, Sun Yat-sen University, Guangzhou city, Guangdong Province, China, 510630.

Corresponding author: Yang Qintai.

E-mail: yang.qt@163.com

Submitted date: 15/8/2013

Accepted date: 26/11/2013
Conclusion: Gammadelta-T-cells and Th17 cells may be involved in the pathogenesis of AR; gammadelta-T-cells may primarily be associated with the secretion of IL-17. (Asian Pac J Allergy Immunol 2014;32:235-9)

Keywords: gammadelta-T-cells, Th17 cells, IL-17, allergic rhinitis

\section{Introduction}

Allergic rhinitis (AR) is caused by exposure to allergens in atopic individuals leading to the IgEmediated release of histamine. Various immune cells and cytokines are involved in non-infectious nasal mucosal and chronic inflammatory diseases. The AR incidence rate has gradually increased in recent years. The disease seriously impacts patient health and quality of life. ${ }^{1}$ It has been reported that T-cell immunity is closely associated with the pathogenesis of $\mathrm{AR}^{2}{ }^{2} \mathrm{~T}$-cells are categorized as $\alpha \beta \mathrm{T}$-cells or $\gamma \delta \mathrm{T}$-cells based on their $\mathrm{T}$-cell receptors (TCR) for antigen recognition. $\alpha \beta T$-cells play an important role in the adaptive immune response, whereas $\gamma \delta \mathrm{T}$-cells are not restricted by the major histocompatibility complex (MHC) and are not dependent on antigen processing and presentation. $\gamma \delta \mathrm{T}$-cells function as a bridge between innate and adaptive immunity and play an important role in the mucosal immune system of the respiratory tract. In addition, $\gamma \delta \mathrm{T}$-cells provide bidirectional immune regulation through the secretion of various cytokines (e.g. IL-17 and interferon-gamma [INF $\gamma]){ }^{3}$ Th17 cells are a new $\alpha \beta$ T-cell subset that plays an important role in promoting inflammation and autoimmune diseases. Th17 cells mainly secrete IL-17 and express ROR $\gamma \mathrm{t}$, which is a key nuclear transcription factor. ${ }^{4,5}$ Our previous studies showed that expression of IL-17 is significantly higher in the peripheral blood of AR patients than in that of the control group, ${ }^{6}$ and patients with persistent AR showed a significant difference in the percentage of peripheral blood $\gamma \delta \mathrm{T}$ cells before and after treatment with specific immunotherapy (SIT). ${ }^{7}$ The aim of this study was to investigate the $\gamma \delta \mathrm{T}$ - and Th17 cells, IL-17 by flow 
cytometry and ELISA in the peripheral blood of AR patients. Our data suggest that $\gamma \delta \mathrm{T}$-cells and Th17 cells may be involved in the pathogenesis of AR.

\section{Methods}

\section{Research subjects}

Blood samples from thirty-two AR patients that received treatment in our department were collected from March 2012 to July 2012. All patients were adults, aged from 18 to 45 years. The patients included 20 males and 12 females with an average age of 29.6 years. The diagnosis of all AR patients met the standard criteria. ${ }^{1}$ Inclusion criteria were moderate/severe allergic rhinitis at least for one year and a skin prick test positive only for the dust mite allergen (ALK, Hørsholm, Denmark). Exclusion criteria were nonallergic inflammatory nasal pathology, chronic sinusitis, asthma and aspirin intolerance, active tuberculosis, an autoimmune disorder, or an immunodeficiency disorder. No patients received systemic or topical corticosteroids, antihistamines or immunotherapy within a month of enrolment. Twenty healthy volunteers (control group, age from 20 to 43 years) from our hospital with no symptoms of allergic rhinitis and negative results in a skin prick test were used in this study, including 9 males and 11 females with an average age of 30.9 years. All subjects had skin prick test for a series of aeroallergens, including dust mite, cockroach, cat hair, dog dander, molds, ragweed, mugwort, pollen. The IRB had approved the research. All participants signed informed consent forms before participation in the experiments.

\section{Instruments and reagents}

Flow cytometry was performed with a BD FACSCalibur ${ }^{\mathrm{TM}}$ model from BD biosciences, USA. Cellquest software (BD Bioscience, USA) was used for data analysis of the flow cytometry. A Bio-Tek ELX-800 microplate reader (Bio-Tek, USA) and the following reagents were also purchased for this study: the $\gamma \delta$ TCR kit (BD Bioscience, USA); PMA (phorbol ester), ionomycin calcium (ionomycin) and BFA (MultiSciences Co.); APC-labeled anti-human CD8 monoclonal antibody (BD Biosciences, USA); PerCP-Cy5.5-labeled anti-human CD3 monoclonal antibody, PE-labeled anti-human IL-17 monoclonal antibody and its matched isotype control (eBioscience Co., USA); fixative and lysis buffer (Invitrogen, USA); and the anti-human IL-17 ELISA kit (eBioscience Co., USA).

\section{Sample collection}

Four-milliliter samples of peripheral blood in heparin solution were collected in the early morning. Two milliliters of blood sample were centrifuged at $300 \mathrm{~g}$ to collect serum for the measurement of the serum IL-17 concentrations and were stored in a $-20^{\circ} \mathrm{C}$ freezer. Two milliliters of the sample were used to perform flow cytometry of $\gamma \delta \mathrm{T}$ cells and Th17 cells within three hours after sample collection.

\section{$\gamma \delta T$ cell assessment by flow cytometry}

Fifty microliters of anticoagulated whole blood was gently vortexed with $20 \mu \mathrm{l}$ of CD3 or $\gamma \delta$ TCR antibody and incubated at room temperature in the dark for 15 minutes. To each tube, $1 \mathrm{ml}$ of 1 xFACS lysis solution was added. The tubes were gently vortexed and incubated at room temperature in the dark for 10 minutes, followed by $300 \mathrm{~g}$ centrifugation for 5 minutes and resuspension in 500 $\mu \mathrm{l}$ of PBS. Immune flow cytometry was then performed and CellQuest software analysis was used for the data analysis.

\section{Th17 cell assessment by flow cytometry}

Two hundred and fifty microliters of peripheral blood was combined with $50 \mu \mathrm{g} / \mathrm{L}$ PMA, 2.0 $\mu \mathrm{mol} / \mathrm{L}$ blocking agent (monensin, Gorky Scientific), and $750 \mu \mathrm{mol} / \mathrm{L}$ ionomycin, and then incubated at $37{ }^{\circ} \mathrm{C}$ in a $5 \% \mathrm{CO}_{2}$ incubator for 4 hours. Cell suspensions were transferred to $1.5 \mathrm{ml}$ EP tubes and centrifuged at $300 \mathrm{~g}$ for 6 minutes. Supernatants were removed and the pellets were washed twice with PBS before performing the flow cytometry analysis. Ten microliters of PECy5-antiCD3 and $10 \mu \mathrm{l}$ of FITC-anti-CD8 were added into the cell suspensions, followed by incubation at room temperature for 30 minutes. After washing with PBS twice, $300 \mu \mathrm{l}$ of fixative was added, and the suspensions were incubated in the dark at $4{ }^{\circ} \mathrm{C}$ for 15 minutes. After centrifugation, the supernatant was removed and cells were washed twice with PBS and aliquoted into two tubes. To each tube $20 \mu \mathrm{PE}-$ anti-IL-17 or $10 \mu \mathrm{l}$ isotype control PE-IgG1 was added, followed by further incubation at room temperature for 30 minutes. After washing twice in PBS, the cells were resuspended in $300 \mu$ and analyzed using a flow cytometer. CellQuest software was used for data analyses. 


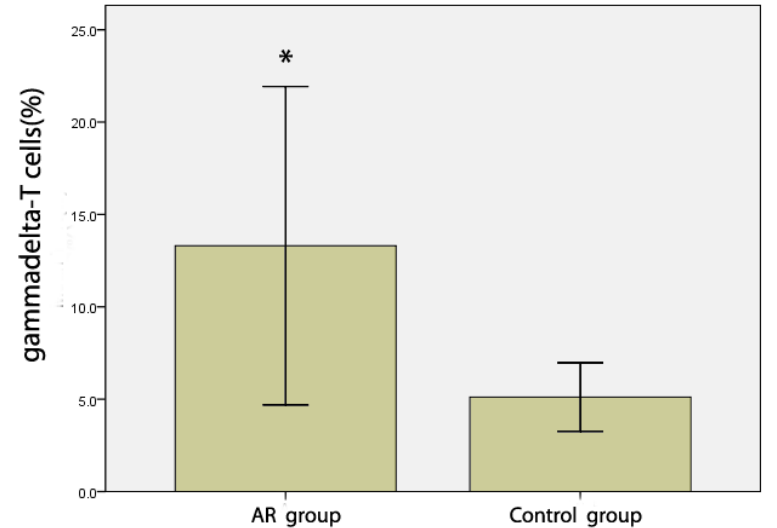

Figure 1. The percentage of $\gamma \delta \mathrm{T}$ cells in the peripheral blood of the AR group and the control group, ${ }^{*}, p<0.01$, compared with the "control group"

\section{ELISA for the measurement of serum IL-17 concentration}

Measurement of IL-17 was done in accordance with the instruction manual of the ELISA kit. The sensitivity of the IL-17 ELISA was determined to be $0.5 \mathrm{pg} / \mathrm{ml}$.

\section{Statistical analysis}

SPSS 16.0 software was used for statistical analysis. Normally distributed data are presented as mean \pm standard deviation $(\chi \pm s)$. Correlations between parameters were analyzed using a Pearson correlation test. $P<0.05$ represents statistically significant differences.

\section{Results}

Flow cytometry analysis of the percentage of $\gamma \delta T$ cell and Th17 cell in the AR and control groups

The percentage of $\gamma \delta \mathrm{T}$-cells in the AR group $(13.30 \pm 8.62 \%)$ was higher than that in the control group $(5.18 \pm 1.86 \%)$, with statistically a significant difference between the groups $(t=5.18 ; P<0.01$; Figure 1).

The percentage of Th17 cells in the peripheral blood of AR patients $(1.86 \pm 0.39 \%)$ was significantly higher than that in the control group $(0.59 \pm 0.26)(t=13.92 ; P<0.01$, Figure 2$)$.

\section{Analysis of the serum IL-17 levels in the AR and control groups}

The serum IL-17 levels in the AR and control groups were $(668.55 \pm 45.15 \mathrm{pg} / \mathrm{ml})$ and $(573.53 \pm$ $17.42 \mathrm{pg} / \mathrm{ml})$, respectively. The IL-17 level in the AR group was significantly higher than that in the control group $(t=8.97 ; P<0.01$, Figure 3$)$.

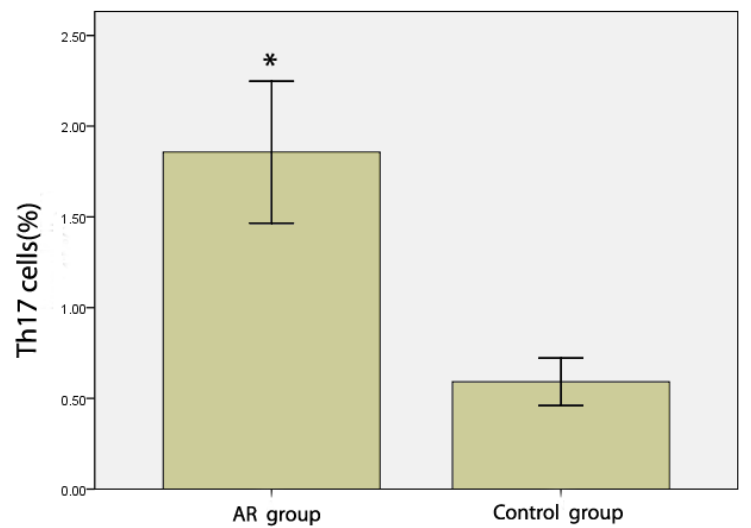

Figure 2. The percentage of Th17 cells in the peripheral blood of the AR group and the control group, *, $p<0.01$, compared with the "control group"

Correlation analyses of the $\gamma \delta T$-cell percentage, Th17 cell percentage and IL-17 levels in the peripheral blood of AR patients

A Pearson correlation analysis showed that the percentages of $\gamma \delta \mathrm{T}$-cells and Th17 cells in the peripheral blood of AR patents were positively correlated $(r=0.597 ; P<0.01$; Figure 4$)$. The percentage of $\gamma \delta$ T-cells and the IL-17 level was also positively correlated $(r=0.469 ; P<0.01$; Figure 25). However, no significant correlation was observed between the IL-17 level and the percentage of Th17 cells $(r=0.100, P<0.05)$.

\section{Discussion}

$\gamma \delta \mathrm{T}$-cells mainly reside in the epidermis and mucosa and account for only $1 \%$ to $10 \%$ of T-cells in the peripheral blood. According to the different combinations of TCR $\gamma$ and $\delta$ chains of the variable (V) region in $\gamma \delta \mathrm{T}$-cells, $\gamma \delta \mathrm{T}$-cells are divided into various subsets which carry different functions. There are two main subgroups of human $\gamma \delta \mathrm{T}$-cells: $\mathrm{V} \delta 2^{+} \mathrm{T}$-cells and $\mathrm{V} \delta 1^{+} \mathrm{T}$-cells. The $\mathrm{V} \delta 2^{+} \mathrm{T}$-cell subset, which primarily includes $\mathrm{V} \gamma 2 / \mathrm{V} \delta 2$ T-cells, accounts for $80 \%$ to $90 \%$ of the total circulating $\gamma \delta \mathrm{T}$-cells, whereas the $\mathrm{V} \delta 1^{+} \mathrm{T}$-cell subset mostly resides in the epithelial mucosa. These subsets share similarities in their receptors and antigens and provide a rapid response to generate large amounts of IL-17 and IFN- $\gamma$ cytokines. ${ }^{3,8}$ In an asthma model, (TCRV $\delta$ knockout mice) it was demonstrated that there was reduced serum $\operatorname{IgE}$ levels and airway reactivity. ${ }^{9}$ Reyes et al. ${ }^{10}$ showed that the absence of $\gamma \delta \mathrm{TCR}$ in animal models of allergic conjunctivitis was associated with reduced clinical symptoms, 


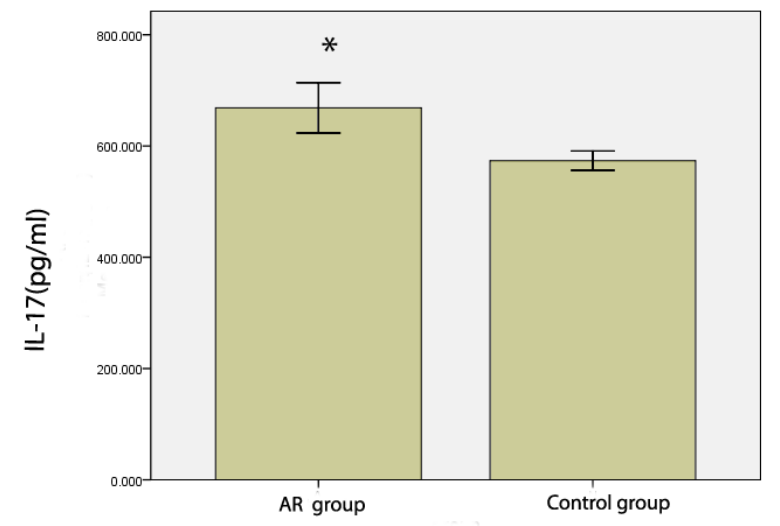

Figure 3. The level of IL-17 cells in the peripheral blood of the AR group and the control group, $*, p<0.01$, compared with the "control group"

eosinophil invasion and Th2 cytokine secretion. $\gamma \delta \mathrm{T}$-cells are increased in the peripheral blood of patients with eczema and dermatitis syndrome ${ }^{[11]}$. Additionally, our previous study showed that patients with persistent AR had a significant difference in the percentage of peripheral blood $\gamma \delta \mathrm{T}$ cells before and after treatment with specific immunotherapy (SIT). After SIT treatment, the $\mathrm{V} \delta 1^{+}$and $\mathrm{V} \delta 2^{+}$subsets of $\gamma \delta \mathrm{T}$-cells were significantly reduced, while the V $\delta 6$ subset of $\gamma \delta \mathrm{T}$ cells increased selectively. ${ }^{7}$ This present study also revealed an increase of $\gamma \delta \mathrm{T}$-cells in the peripheral blood of AR patients, suggesting that $\gamma \delta \mathrm{T}$-cells may be involved in the inflammation of $\mathrm{AR}$, and further study for the function of subset of $\gamma \delta \mathrm{T}$-cells in AR would be worthwhile.

Studies have demonstrated that $\gamma \delta \mathrm{T}$-cells promote a Th17 cell response after administration of complete Freund's adjuvant in mice, ${ }^{12}$ and IL-17 (+) $\gamma \delta \mathrm{T}$-cells enhance Th17 cell differentiation in T-cell mediated colitis. ${ }^{13}$ Ex-vivo PBMNC evaluation has shown that allergic patients have higher frequencies of IL-17 producing $\mathrm{T}$ cells. ${ }^{14}$ The results of this study also showed that the percentage of Th17 cell in AR was significantly higher than the control group and there was a positive correlation between the Th17 percentage and $\gamma \delta \mathrm{T}$-cells. These results suggest that Th17 cell may be involve in AR pathogenesis and that $\gamma \delta \mathrm{T}$-cells may have an immunomodulatory function on Th17 cells in the peripheral blood of AR patients.

IL-17 is an important effector of Th17 cells with a strong pro-inflammatory role in rheumatoid arthritis. Increased expression of IL-17 has been observed in multiple sclerosis, asthma, systemic

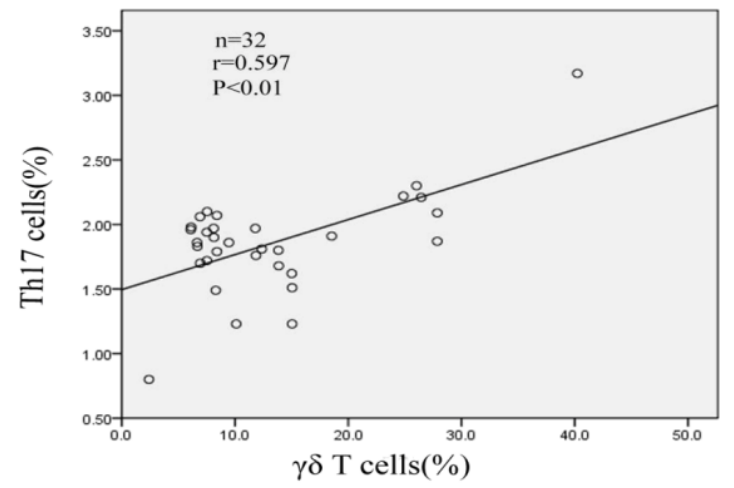

Figure 4. Scatter plot of the percentages of $\gamma \delta \mathrm{T}$ cells and Th17 cells in the peripheral blood of AR patients.

lupus erythematosus and transplant rejection. ${ }^{15} \mathrm{IL}$ 17 may play a pathogenic role in an established AR mouse model. ${ }^{16,17}$ Expression of IL-17 is significantly higher in the peripheral blood of AR patients than in the control group. ${ }^{6}$ In a study by Ciprandi et al., ${ }^{18}$ a positive correlation was demonstrated between IL-17 expression in the peripheral blood of AR patients and the severity of AR clinical symptoms. Nasal allergen challenge induces the enhanced secretion of IL-17 in the lower airways of nonasthmatic patients with AR. ${ }^{19}$ Studies in recent years have shown that $\gamma \delta \mathrm{T}$-cells are an important source of IL-17 production. In infectious disease models of Listeria monocytogenes ${ }^{20}$ and Bacillus subtilis-induced hypersensitivity pneumonitis, $^{21} \gamma \delta \mathrm{T}$-cells rapidly produces large amounts of IL-17 after the dendritic cells produce IL-23 following infection. Our results demonstrate an increased level of IL-17 and Th17 cells in the peripheral blood of AR patients. Increased levels of IL-17 showed a positive correlation with $\gamma \delta \mathrm{T}$-cells but no significant correlation with Th17 cells, suggesting that IL-17 in the peripheral blood of AR may be primarily generated by the $\gamma \delta \mathrm{T}$-cells. Further in-depth research is needed to determine which subset of $\gamma \delta \mathrm{T}$-cells is involved in the secretion of IL17.

In conclusion, the present study showed higher expression of $\gamma \delta \mathrm{T}$-cells, Th17 cells and IL-17and positive correlations between Th17 cells, IL-17 level and $\gamma \delta \mathrm{T}$-cells in the peripheral blood of AR patients. This study provides preliminary evidence that $\gamma \delta \mathrm{T}$ cells and Th17 cells may be involved in the pathogenesis of AR. More studies with a large sample size are required to confirm the role of $\gamma \delta \mathrm{T}$ - 


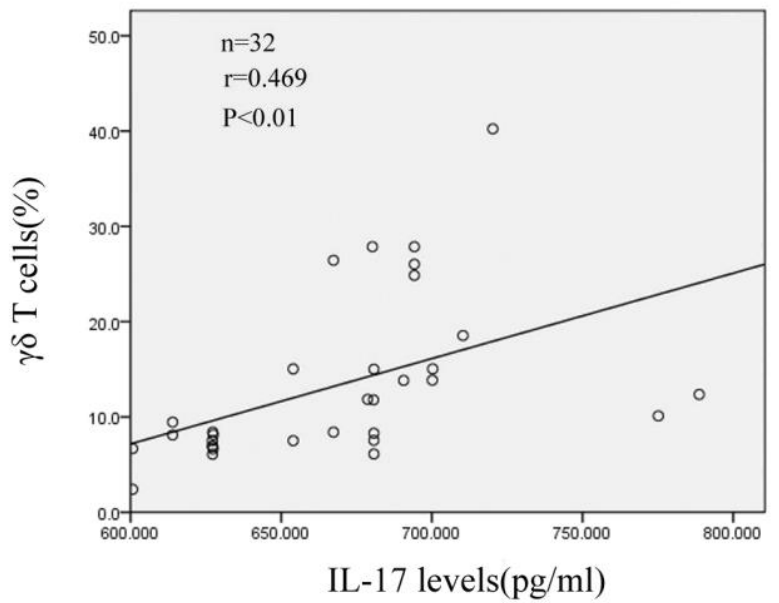

Figure 5. Scatter plot of the percentages of $\gamma \delta \mathrm{T}$ cells and IL-17 levels in the peripheral blood of AR patients.

cells and Th17 cells in the AR and assess IL-17 expression at a cell level.

\section{Acknowledgement}

This study was supported by Natural Science Foundation of Guangdong Province (S2013010016386), National Natural Science Foundation of China (81371072).

\section{References}

1. Bousquet J, Khaltaev N, Cruz AA, Denburg J, Fokkens WJ, Togias A, et al. Allergic rhinitis and its impact on asthma (ARIA) 2008 Update (in collaboration with the World Health Organization, GA2LEN and AllerGen). Allergy. 2008;63(Suppl 86):8-160.

2. Romagnani S. Regulation of the T cell response. Clin Exp Allergy. 2006;36:1357-66.

3. Chien HY, Bonneville M. gamma delta T cell receptors. Cell Mol Life Sci. 2006;63:2089- 94.

4. Harrington LE, Hatton RD, Mangan PR, Turner H, Murphy TL, Murphy KM, et al. Interleukin 17-producing CD4+ effector T cells develop via a lineage distinct from the $\mathrm{T}$ helper type 1 and 2 lineages. Nat Immunol. 2005;6:1123-32.

5. Ivanov II, McKenzie BS, Zhou L, Tadokoro CE, Lepelley A, Lafaille JJ, et al. The orphan nuclear receptor RORgammat directs the differentiation program of proinflammatory IL-17+ T helper cells. Cell. 2006;126:1121-33.

6. Xuekun Huang, Qintai Yang, Yulian Chen, Peng Li, Gehua Zhang, Yuan Li. Expressions of IL-17, IL-21 and IL-23 in the serum of allergic rhinitis patients . J Med Biochem. 2011;30:32327.
7. Yang QT, Huang XK, Li P, Chen YL, Zhang GH. Analyses of subfamily lineage and cloning on the peripheral blood $\mathrm{T}$ cell antigen recognition receptor $\mathrm{V} \gamma$ and $\mathrm{V} \delta$ before and after the immunotherapy of allergic rhinitis. The Chinese Journal of otolaryngology head and neck surgery. 2011,46:992-97.

8. Girardi M. Immunosurveillance and immunoregulation by gammadelta T cells . J Invest Dermatol. 2006;126:25- 31.

9. Schramm CM, Puddington L, Yiamouyiannis CA, Lingenheld EG, Whiteley HE, Wolyniec WW, et a1. Proinflammatory roles of Tcell receptor(TCR) $\gamma \delta$ and TCR $\alpha \beta$ lymphocytes in a murine model of asthma. Am J Respir Cell Mol Biol. 2000;22:218-25.

10. Reyes NJ, Mayhew E, Chen PW, Niederkorn JY. $\gamma \delta$ T cells are required for maximal expression of allergic conjunctivitis. Invest Ophthalmol Vis Sci. 2011;52:2211-16.

11. Cairo C, Arabito E, Landi F, Casati A, Brunetti E, Mancino G, et al. Analysis of circulating gammadelta $T$ cells in children affected by IgE-associated and non-IgE-associated allergic atopic eczema/dermatitis. Clin Exp Immunol. 2005;141:116-21.

12. Roark CL, Huang Y, Jin N, Aydintug MK, Casper T, Sun D, et al. A canonical $\mathrm{V} \gamma 4 \mathrm{~V} \delta 4+\gamma \delta \mathrm{T}$ cell population with distinct stimulation requirements which promotes the Th17 response. Immunol Res. 2013;55:217-30.

13. Do JS, Visperas A, Dong C, Baldwin WM 3rd, Min B. Cutting edge: Generation of colitogenic Th17 CD4 T cells is enhanced by IL-17+ $\gamma \delta$ T cells. J Immunol. 2011;186:4546-50.

14. Ciprandi G, Filaci G, Battaglia F, Fenoglio D.Peripheral Th-17 cells in allergic rhinitis: New evidence. Int Immunopharmacol. 2010;10:226-9.

15. Iwakura Y, Ishigame H.The IL-23/IL-17 axis in inflammation. J Clin Invest, 2006;116:1218-22.

16. Quan SH, Zhang YL, Han DH, Iwakura Y, Rhee CS. Contribution of interleukin 17A to the development and regulation of allergic inflammation in a murine allergic rhinitis model. Ann Allergy Asthma Immunol. 2012;108:342-50.

17. Wang M, Zhang W, Shang J, Yang J, Zhang L, Bachert C. Immunomodulatory effects of IL-23 and IL-17 in a mouse model of allergic rhinitis. Clin Exp Allergy. 2013;43:956-66.

18. Ciprandi G, De Amici M, Murdaca G, Fenoglio D, Ricciardolo F, Marseglia G, et al. Serum interleukin-17 levels are related to clinical severity in allergic rhinitis . Allergy. 2009;64:1375-78.

19. Semik-Orzech A, Barczyk A, Wiaderkiewicz R, Pierzchala W.Interleukin 17 and RANTES levels in induced sputum of patients with allergic rhinitis after a single nasal allergen challenge. Ann Allergy Asthma Immunol. 2009;103:418-24.

20. Hamada S, Umemura M, Shiono T, Tanaka K, Yahagi A, Begum $\mathrm{MD}$, et al. IL-17A produced by gammadelta $\mathrm{T}$ cells plays a critical role in innate immunity against listeria monocytogenes infection in the liver. J Immunol. 2008;181:3456-63.

21. Simonian PL, Roark CL, Born WK, O'Brien RL, Fontenot AP. Gammadelta $\mathrm{T}$ cells and Th17 cytokines in hypersensitivity pneumonitis and lung fibrosis. Transl Res. 2009;154:222- 27. 